\title{
Elliptic Membership Functions and the Modeling Uncertainty in Type-2 Fuzzy Logic Systems As Applied to Time Series Prediction
}

\author{
Erdal Kayacan*, Simon Coupland ${ }^{\dagger}$, Robert John ${ }^{\ddagger}$ and Mojtaba Ahmadieh Khanesar $§$ \\ ${ }^{*}$ School of Mechanical and Aerospace Engineering, Nanyang Technological University, 639798, Singapore \\ E-mail: erdal@ntu.edu.sg \\ ${ }^{\dagger}$ School of Computer Science and Informatics, De Montfort University, The Gateway, Leicester, LE1 9BH, UK \\ E-mail: simonc@dmu.ac.uk \\ ${ }^{\ddagger}$ Lab for Uncertainty in Data and Decision Making (LUCID), School of Computer Science, University of Nottingham, \\ Nottingham, NG8 1BB, UK, E-mail: Robert.John@ nottingham.ac.uk

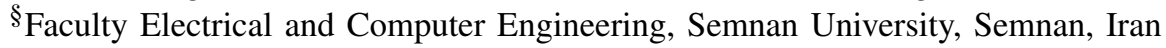 \\ E-mail: ahmadieh@semnan.ac.ir
}

\begin{abstract}
In this paper, our aim is to compare and contrast various ways of modeling uncertainty by using different type-2 fuzzy membership functions available in literature. In particular we focus on a novel type-2 fuzzy membership function, - "Elliptic membership function". After briefly explaining the motivation behind the suggestion of the elliptic membership function, we analyse the uncertainty distribution along its support, and we compare its uncertainty modeling capability with the existing membership functions. We also show how the elliptic membership functions perform in fuzzy arithmetic. In addition to its extra advantages over the existing type- 2 fuzzy membership functions such as having decoupled parameters for its support and width, this novel membership function has some similar features to the Gaussian and triangular membership functions in addition and multiplication operations. Finally, we have tested the prediction capability of elliptic membership functions using interval type-2 fuzzy logic systems on US Dollar/Euro exchange rate prediction problem. Throughout the simulation studies, an extreme learning machine is used to train the interval type-2 fuzzy logic system. The prediction results show that, in addition to their various advantages mentioned above, elliptic membership functions have comparable prediction results when compared to Gaussian and triangular membership functions.

Index Terms-Elliptic membership function, type-2 fuzzy logic theory, uncertainty, fuzzy sets, Gaussian, triangular, time series prediction.
\end{abstract}

\section{INTRODUCTION}

Transition from type-1 to type- 2 is still a hot topic in fuzzy logic community as it is believed that type-2 fuzzy logic systems (T2FLSs) appear to be a more promising method than their type- 1 counterparts for handling real world applications, which exhibit measurement noise, uncertainties such as noisy data and changing environments [1]-[3]. Regardless of being type-1 or type-2, membership functions (MFs) are inevitable components of any fuzzy logic system which were introduced by Zadeh in his paper on fuzzy sets in 1965. The basic definitions of fuzzy logic and MFs of Lotfi A. Zadeh were as follows:
"A fuzzy set is a class of objects with a continuum of grades of membership. Such a set is characterized by a membership (characteristic) function which assigns to each object a grade of membership ranging between zero and one." [4].

L. A. Zadeh

A type-2 fuzzy set is where the membership grade is not in $[0,1]$ but is itself a (type-1) fuzzy set. Zadeh introduced the notion of type-n fuzzy sets.

Definition: Type- $n$ fuzzy sets [5]. A fuzzy set is of type $n, n=$ $2,3, \ldots$, if its membership function ranges over fuzzy sets of type $n-l$. The membership function of a fuzzy set of type 1 ranges over the interval $[0,1]$.

This elegant definition provides an excellent, concise description of type-n fuzzy sets. It is worth noting here that, to date, researchers are working with type- 1 and type-2 fuzzy sets with limited research into type-n fuzzy sets [6].

Now we introduce a more modern definition:

Definition: A Type-2 Fuzzy Set [7] is a bivariate function on the cartesian product $X \times[0,1]$ into $[0,1]$. In other words, $\mu$ on the universe of discourse $X$ is given by $u: X \times[0,1] \rightarrow[0,1]$.

This paper is concerned with interval type-2 fuzzy sets.

Definition: An interval type-2 fuzzy set is where the type-1 fuzzy set in the secondary takes value unity everywhere. That is an Interval type-2 fuzzy set is a function from $X$ into $D[0,1]$ where $D[0,1]$ is the set of closed sub-intervals in $[0,1][7]$.

An interval type-2 fuzzy set is fully defined by it's Footprint of Uncertainty (FOU).

Definition [7]: The FOU is the union of all the primary memberships and is given by $\{(x, t) \in X \times[0,1] \mid \mu(x, t)>0\}$. As stated in [7] it is the support of $\mu$.

In the type-2 fuzzy logic research community we characterise type-2 fuzzy MFs by the shape of the FOU. There exist a number of type-2 fuzzy MFs in literature, i.e. triangular, trapezoidal, Gaussian, two-sided Gaussian, Bell-shaped, sigmoid, pi-shaped, etc. However, all the aforementioned MFs 
have an adverse common feature: the parameters responsible for the support and the width are coupled. On the other hand, elliptic MFs have certain values on the left and right end of the support, and it deals with uncertainty on the rest of the support. By designing such a novel type-2 fuzzy MF, the parameters responsible for the width of uncertainty are de-coupled from the parameters responsible for the center and the support of the MF. This feature allows us to analyze how the uncertainty in the input distorts the inference of the T2FLS [8].

Even if there are some fuzzy control software packages, which allow the user to design custom shaped MFs, there is no systematic way to choose a fuzzy MF in order to achieve a better control performance. In this paper, our aim is to compare and contrast the different ways of modeling uncertainty by using different type-2 MFs in literature. In particular, we focus on a novel type-2 MF, namely "Elliptic MF". In our analysis, we look at the uncertainty modeling capability of different type-2 MFs and make some comments about fuzzy arithmetic, by using the novel elliptic MF. All these analysis shows that the novel MF is promising in terms of its uncertainty modeling capability.

The rest of the paper is organized as follows: In Section II, we go over both the existing type-2 MFs and elliptic MF. In Section III, we give a brief critique for their capability of modeling uncertainty. Section IV presents fuzzy arithmetic by using different type- 2 MFs, and the prediction capability of elliptic membership functions is illustrated in Section V. Finally, some conclusions are drawn from this study in Section VI.

\section{EXISTing TyPe-2 MFs IN THE Literature}

Gaussian type MFs are commonly preferred in literature which are differentiable along the support which is advantageous especially when designing parameter update rules for adaptive neuro-fuzzy systems. Another prominent feature of these MFs is that they are always continuous, whereas some other MFs are continuous only under certain conditions. In Figs. 1(a)-1(b), Gaussian type-2 fuzzy sets with uncertain standard deviation and uncertain mean are shown, respectively. The mathematical expression for the Gaussian MF is expressed as:

$$
\tilde{\mu}(x)=\exp \left(-\frac{1}{2} \frac{(x-c)^{2}}{\sigma^{2}}\right)
$$

where $c$ and $\sigma$ are the center and the width of the MF, $x$ is the input vector.

In Figs. 1(c)-1(d), triangular type-2 fuzzy sets with uncertain width and uncertain center are shown. The mathematical expression for the MF is expressed as:

$$
\tilde{\mu}(x)=\left\{\begin{array}{cc}
1-\frac{|x-c|}{d} & \text { if } c-d<x<c+d \\
0 & \text { else }
\end{array}\right.
$$

where $c$ and $d$ are the center and the width of the MF, $x$ is the input vector.

Which MF to deploy in a real application is often chosen by trial and error or by preference. There is still a lot of research need as to which type- 2 MFs are best for which

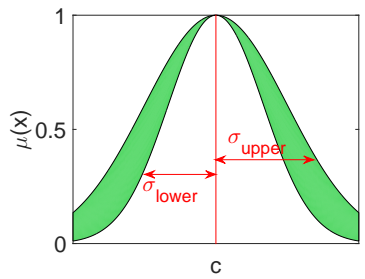

(a)

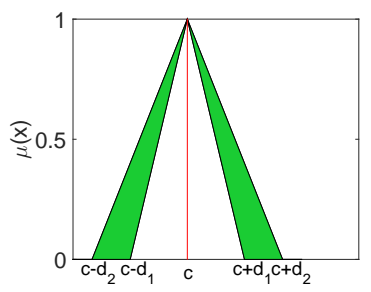

(c)

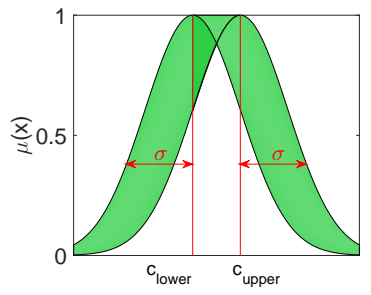

(b)

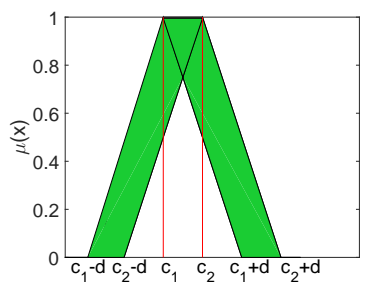

(d)
Fig. 1. Type-2 fuzzy Gaussian and triangular MFs: uncertain standard deviation (a), uncertain mean (b), uncertain width (c) and uncertain center (d)

application. In [9], a detailed analysis has been done on Gaussian and trapezoidal MFs in interval type-2 fuzzy logic controllers. It has been reported that interval type-2 fuzzy logic controllers by using Gaussian MFs are simpler in design, always continuous and faster for small rulebases, whereas the controllers by using trapezoidal MFs are simpler in analysis [9].

In this paper we are interested in a new and novel type-2 MF - the elliptic MF [8]. These MFs have certain values on the left and right end of the support, and it deals with uncertainty on the rest of the support. Inspired by the formulation of the triangular MFs, the lower and the upper MFs are defined with the following formulas:

$$
\begin{aligned}
& \bar{\mu}(x)=\left\{\begin{array}{cc}
\left(1-\left|\frac{x-c}{d}\right|^{a_{1}}\right)^{1 / a_{1}} & \text { if } c-d<x<c+d \\
0 & \text { else }
\end{array}\right. \\
& \underline{\mu}(x)=\left\{\begin{array}{cc}
\left(1-\left|\frac{x-c}{d}\right|^{a_{2}}\right)^{1 / a_{2}} & \text { if } c-d<x<c+d \\
0 & \text { else }
\end{array}\right.
\end{aligned}
$$

where $c$ and $d$ are the center and the width of the MF, $x$ is the input vector. The parameters $a_{1}$ and $a_{2}$ determine the width of the uncertainty of the proposed MF, and these parameters should be selected in the following form:

$$
\begin{aligned}
a_{1} & >1 \\
0<a_{2} & <1
\end{aligned}
$$

Figures 2(a), 2(b), 2(c) and 2(d) show the shapes of the proposed MF for $a_{1}=a_{2}=1, a_{1}=1.2, a_{2}=0.8, a_{1}=1.4, a_{2}=$ 0.6 and $a_{1}=1.6, a_{2}=0.4$, respectively. As can be seen from Fig. 2(a), the shape of the proposed type-2 MF is changed to a type- 1 triangular MF when its parameters are selected as $a_{1}=a_{2}=1$. 


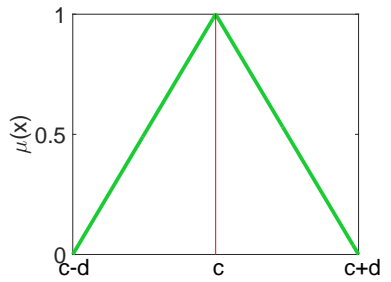

(a)

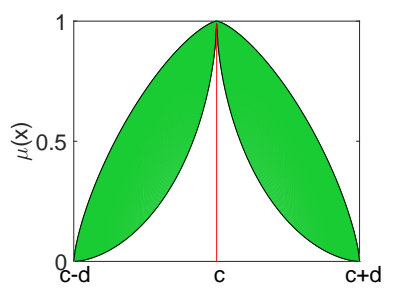

(c)

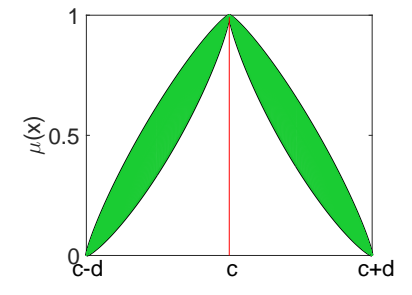

(b)

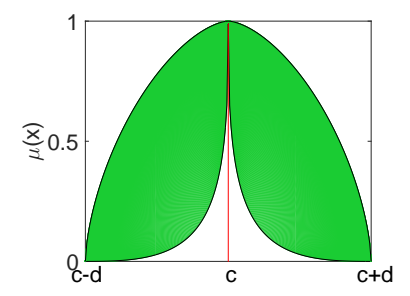

(d)
Fig. 2. The shape of the proposed type-2 MF for $a_{1}=1$ and $a_{2}=2$ (a) for $a_{1}=1.2$ and $a_{2}=0.8(\mathrm{~b})$, for $a_{1}=1.4$ and $a_{2}=0.6(\mathrm{c})$, for $a_{1}=1.6$ and $a_{2}=0.4(\mathrm{~d})$

The elliptic type-2 fuzzy MF has already been used for identification [10] and control [11] purposes. A similar MF to the elliptic MF, a diamond-shaped type-2 fuzzy MF, was initially proposed [12]. It has similar features such as to have certain values both on the left and right end points of the support and to represent uncertainty on the rest of the support. However, elliptic MF is smoother than the diamond-shaped type-2 fuzzy MF and is differentiable in more points of its support set than diamond-shaped type-2 MF. Moreover, this type of type-2 MFs has more parameters to tune than elliptic type-2 MF which make it hard to tune.

\section{UnCERTAinty Modeling CAPABility}

In this section we consider all these sets form the perspective of uncertainty modeling. This is, by its very nature, somewhat subjective but we hope is interesting for the reader.

\section{A. Uncertainty Distribution Along the Support}

The capability of modeling uncertainty plays a vital role not only in control systems but also in decision support systems. In particular, fuzzy decision making systems are designed in a way that they will be able to model the uncertainty resulting a strong and accurate decision support system. Unfortunately, as already stated in the previous subsection, there is no systematic way to choose a fuzzy $\mathrm{MF}$ in order to achieve a better uncertainty modeling capability. In this subsection, our aim is to compare and contrast the different ways of modeling uncertainty for different type-2 MFs in literature.

1) Critique for Gaussian and Triangular MFs: Let us imagine a horizontal line on Figs. 1(a) and 1(c). When the horizontal line moves from the most top point, which corresponds to $\mu(x)=1$, to the most bottom point, which corresponds to $\mu(x)=0$, the uncertainty on the input will increase for a specific membership value. In other words, whereas the uncertainty is less around the center, we are more uncertain when we are far away from the center. The former part is reasonable as the expert is probably more precise around the center of the MF, however, it is being more ambiguous along the support as being far away from the center. The latter part is not realistic from a human expert point of view, because if an expert is precise around the center that a specific input belongs to a fuzzy set, the same expert must be also precise that after some region along the support, the input will definitely not belong to this specific set. In this case, it is not reasonable to increase the uncertainty constantly.

As can be seen from Figs. 1(b) and 1(d), the uncertainty is the same along the support. This type of modeling is not reasonable as the expert cannot be equally uncertain along the support. As he/she is the person who places the MF on a specific center value, we expect him/her to be more or less uncertain on the specific places on the support.

2) Critique for Elliptic MFs: As can be seen from Figs. 2(b) and 2(c), the expert is very precise that a specific input around the center will belong to a specific fuzzy set. As we are moving from the center towards the left or right end point of the support $(c+d$ or $c-d)$, he/she is less certain about his/her decision. It is interesting that after the half way, the expert starts being more precise that a specific input does not belong this fuzzy set. The reason for such a modeling is explained in the next paragraph with the help of Fig. 3.

In Fig. 3, having more than one MF case is illustrated. There are two elliptic type- 2 fuzzy MFs; whereas one of which is centered at 0 and the other is centered at 1 . The expert is quite precise that the first input, 0.1 , belongs to the magenta fuzzy set. However, the expert is again precise that the third input, 0.9, does not belong to the magenta fuzzy set. On the other hand, the expert is again precise that the third input, 0.9, belongs to the green fuzzy set whereas he/she is again precise that the first input, 0.1, does not belong to the green fuzzy set. The expert is most uncertain around 0.5. We believe that this way of uncertainty representation is closer to the human way of thinking when compared to Gaussian or triangular MFs.

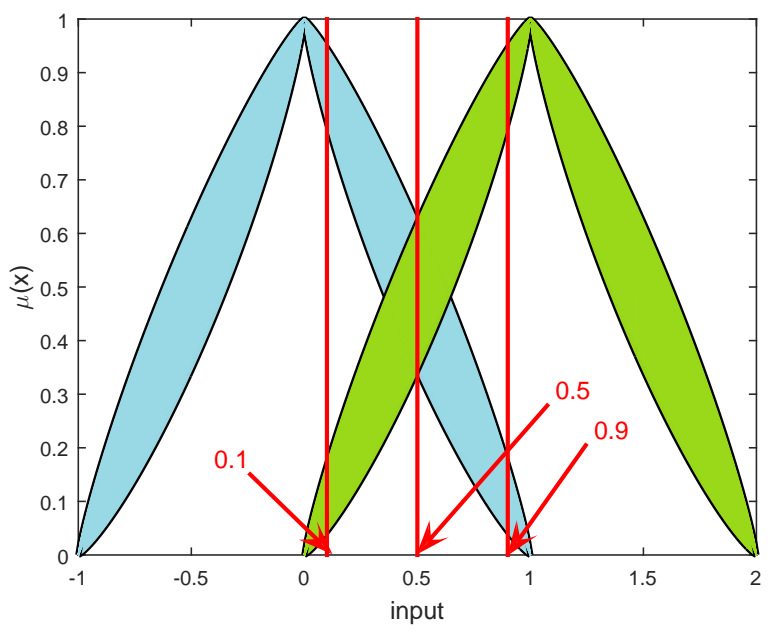

Fig. 3. Having more than one Elliptic MF 


\section{ARithmetic Using Different Type-2 FuZzy MFs}

In this Section we consider how uncertainty is propagated through two arithmetic operation: addition and multiplication.

\section{A. Addition}

It is well understood that addition can be performed on type1 fuzzy sets through applying addition to the parameters of the type-1 fuzzy sets. We now show that this is also the case for triangular, Gaussian and elliptic interval type-2 fuzzy sets.

The addition of two type- 1 fuzzy sets $A$ and $B$ is given in (6) where values of $x, y$ and $z$ all belong to the same numeric domain. Since all operations on an interval type-2 fuzzy can be defined by operations on the upper and lower MFs as defined by (7) and (8).

$$
\begin{aligned}
& \mu_{A+B}(z)=\underset{\forall z=x+y}{\operatorname{argmax}}\left(\mu_{A}(x) \wedge \mu_{B}(y)\right) \\
& \mu_{\underline{A \tilde{+} B}}(z)=\underset{\forall z=x+y}{\operatorname{argmax}}\left(\mu_{\tilde{\tilde{\tilde{A}}}}(x) \wedge \mu_{\underline{\tilde{\tilde{\tilde{U}}}}}(y)\right) \\
& \mu_{\overline{A \tilde{+} B}}(z)=\underset{\forall z=x+y}{\operatorname{argmax}}\left(\mu_{\tilde{\tilde{A}}}(x) \wedge \mu_{\tilde{\tilde{B}}}(y)\right)
\end{aligned}
$$

We now perform addition on three pairs of broadly equivalent triangular, Gaussian and elliptic interval type-2 fuzzy sets. Table I gives the parameters of the these set and the expected parameters of the sets resulting from the addition of these sets. These parameters are taken from the Authors previous work on addition of type-2 fuzzy sets [13]-[15].

TABLE I

PARAmeters of Sets Undergoing AdDition

\begin{tabular}{|c|c|c|c|c|c|c|c|c|c|}
\hline Type & \multicolumn{3}{|c|}{ About 3} & \multicolumn{3}{c|}{ About 12} & \multicolumn{3}{c|}{ About 15} \\
\hline Triangular $(\underline{s}, m, \underline{e})$ & 1.5 & 3 & 4.5 & 10.5 & 12 & 13.5 & 12 & 15 & 18 \\
\hline Triangular $(\bar{s}, m, \bar{e})$ & 1 & 3 & 5 & 10 & 12 & 14 & 11 & 15 & 19 \\
\hline Gaussian $(c, \underline{\sigma})$ & 3 & \multicolumn{2}{|c|}{0.5} & 12 & \multicolumn{2}{|c|}{0.5} & 15 & \multicolumn{2}{|c|}{1.0} \\
\hline Gaussian $(c, \bar{\sigma})$ & 3 & \multicolumn{2}{|c|}{0.8} & 12 & \multicolumn{2}{|c|}{0.8} & 15 & \multicolumn{3}{|c|}{1.6} \\
\hline Elliptic $\left(c, d, a_{1}\right)$ & 3 & 2 & 0.8 & 12 & 2 & 0.8 & 15 & 4 & 0.8 \\
\hline Elliptic $\left(c, d, a_{2}\right)$ & 3 & 2 & 1.2 & 12 & 2 & 1.2 & 15 & 4 & 1.2 \\
\hline
\end{tabular}

Figures 4, 5 and 6 depict these addition operations of the respective three forms of MF; triangular, Gaussian and elliptic. The relationship between the parameters under addition is quite straight forward. To add a pair of triangular or Gaussian interval fuzzy numbers we simply add their respective parameters. This is a useful property which is well known for triangular and Gaussian fuzzy sets and here we will now show an equivalent property for elliptic fuzzy sets.

The parameters of an elliptic fuzzy set $\tilde{C}$ can be calculated from from the two elliptic fuzzy sets $\tilde{A}$ and $\tilde{B}$ whose addition it results from. The following equations given this relation ship where $a_{1}, a_{2}, c$ and $c$ are set parameters and the subscripts denote which set the parameters belong to.

$$
\begin{gathered}
c_{\tilde{C}}=c_{\tilde{A}}+c_{\tilde{B}} \\
d_{\tilde{C}}=d_{\tilde{A}}+d_{\tilde{B}} \\
a 1_{\tilde{A}}=a 1_{\tilde{B}}=a 1_{\tilde{C}} \\
a 2_{\tilde{A}}=a 2_{\tilde{B}}=a 2_{\tilde{C}}
\end{gathered}
$$

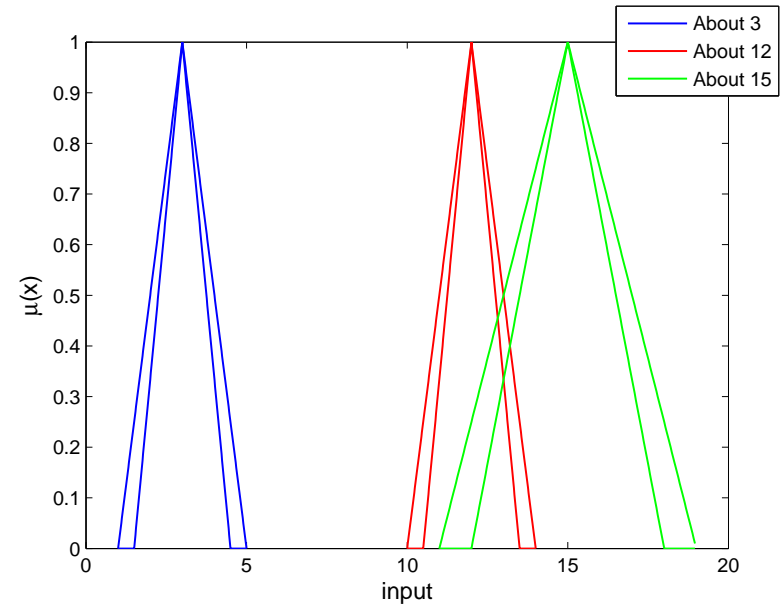

Fig. 4. The addition of two triangular interval fuzzy sets About 3 and About 12 .

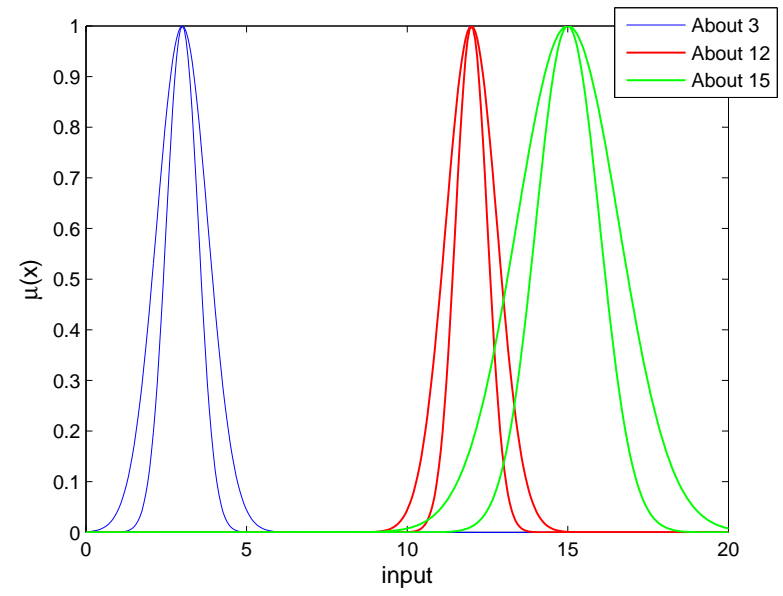

Fig. 5. The addition of two Gaussian interval fuzzy sets About 3 and About 12 .

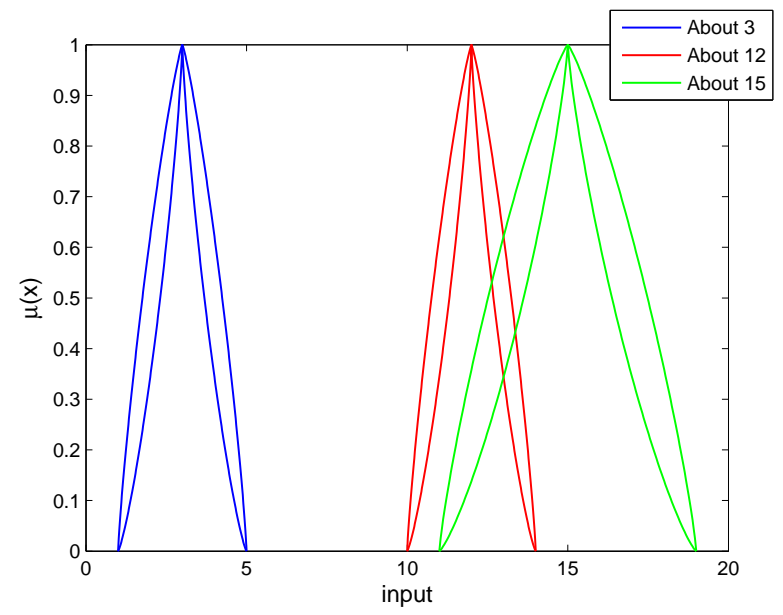

Fig. 6. The addition of two Elliptic interval fuzzy sets About 3 and About 12. 


\section{B. Multiplication}

Multiplication of fuzzy numbers of any kind is a more complex operation than addition. Typically, although not exclusively, multiplication is performed via $\alpha$-cuts as given by (13).

$$
C=\forall_{\alpha \in[0,1]} A_{\alpha} \times B_{\alpha}
$$

For continuous fuzzy sets $A_{\alpha}$ and $B_{\alpha}$ are intervals and therefore interval arithmetic may be used. For discrete fuzzy sets $A_{\alpha}$ and $B_{\alpha}$ are crisp sets, however only minimum and maximum values in these sets need be multiplied. As with addition multiplication of type-2 interval fuzzy sets can be performed on the upper and lower MFs independently as given in (14) and (15).

$$
\begin{aligned}
& \underline{C}=\forall_{\alpha \in[0,1]} \underline{A}_{\alpha} \times \underline{B}_{\alpha} \\
& \bar{C}=\forall_{\alpha \in[0,1]} \bar{A}_{\alpha} \times \bar{B}_{\alpha}
\end{aligned}
$$

We now perform multiplication on three pairs of broadly equivalent triangular, Gaussian and elliptic interval type-2 fuzzy sets. Table II gives the parameters of the these sets. These parameters are taken from the Authors previous work on addition of type-2 fuzzy sets [13]-[15].

Figures 7, 8 and 9 depict these multiplication operations of the respective three forms of MF; triangular, Gaussian and elliptic using 10,000 $\alpha$-cuts. Unlike addition, for multiplication of two fuzzy numbers does not yield a fuzzy set which can be easily constructed from it's parameters. However, the parameters of the two sets being added can give a fuzzy set with a new set of parameters which approximate the result from multiplication.

For triangular and elliptic type-2 interval MFs the relevant parameters can be calculated. For triangular MFs all parameters are multiplied and this yields the set depicted in Fig. 7. For elliptic the centres $(C)$ are multiplied together and the widths are given by (16).

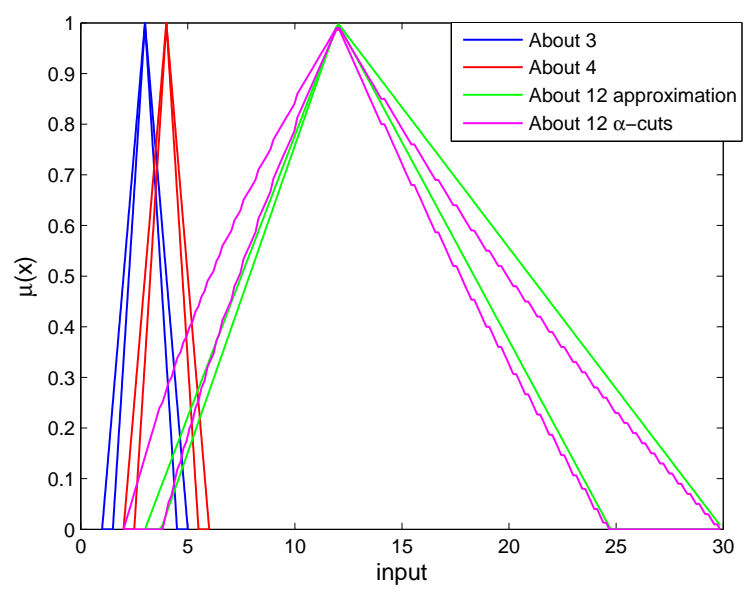

Fig. 7. The multiplication of two triangular interval fuzzy sets About 3 and About 4.

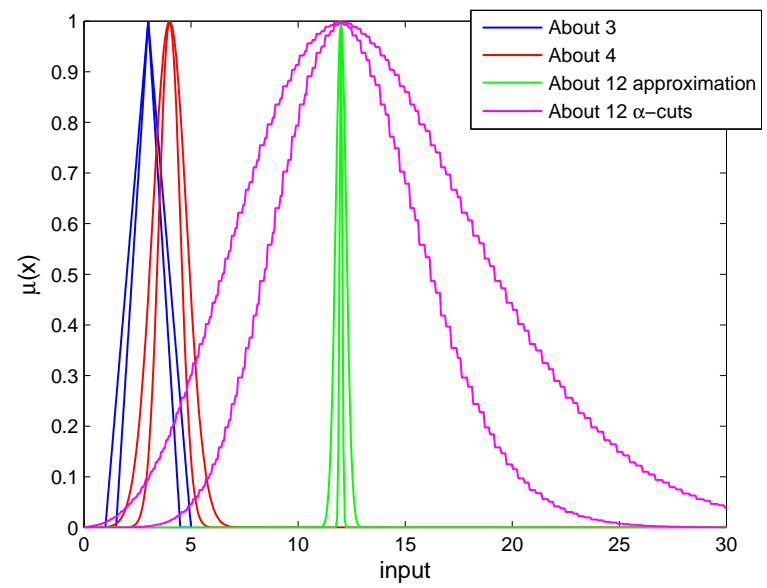

Fig. 8. The multiplication of two Gaussian interval fuzzy sets About 3 and About 4.

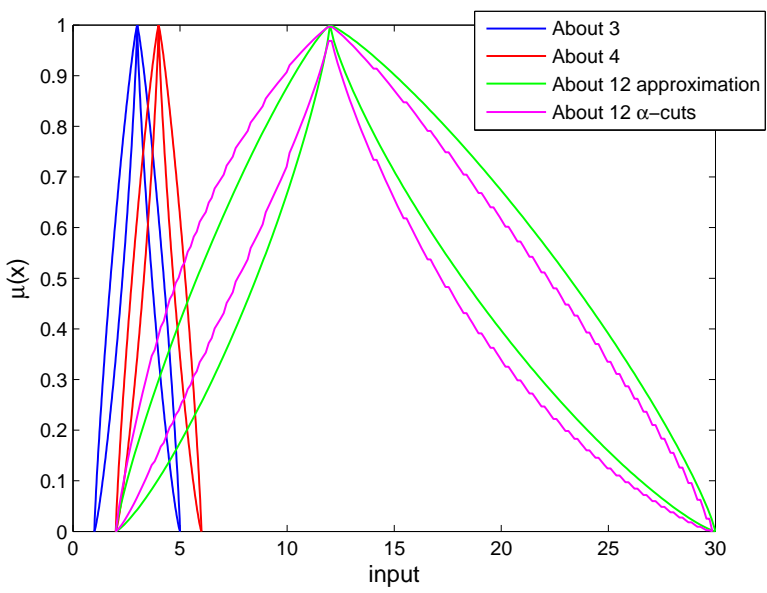

Fig. 9. The multiplication of two Elliptic interval fuzzy sets About 3 and About 4.

$$
d_{\tilde{C}}(x)= \begin{cases}c_{\tilde{C}}-\left(c_{\tilde{A}}-d_{\tilde{A}}\right) \times\left(c_{\tilde{B}}-d_{\tilde{B}}\right) & \text { if } x \leq c_{\tilde{C}} \\ \left(c_{\tilde{A}}-d_{\tilde{A}}\right) \times\left(c_{\tilde{B}}-d_{\tilde{B}}\right)-c_{\tilde{C}} & \text { if } x>c_{\tilde{C}}\end{cases}
$$

From Figs. 7, 8 and 9 it is clear that it is possible to approximate the multiplication operation quite easily with triangular and elliptic fuzzy set but not with Gaussian.

\section{Application to the Prediction of USD/Euro EXCHANGE RATE}

In this section, the capability of IT2FLS with elliptic type$2 \mathrm{MF}$ in the prediction of US Dollar/Euro exchange rate is investigated. The data set used belongs to the period of $30-O c t-1998$ to $16-A u g-2016$. The data set is normalized to the interval of $[0,1]$ and converted to the true values after prediction. The main intention of this section is to show that the prediction performance of interval type-2 fuzzy logic systems (IT2FLSs) with elliptic type-2 MF is comparable and 
TABLE II

Parameters of Sets Undergoing Multiplication

\begin{tabular}{|c|c|c|c|c|c|c|c|c|c|}
\hline Type & \multicolumn{3}{|c|}{ About 3 } & \multicolumn{3}{c|}{ About 4 } & \multicolumn{3}{c|}{ About 12} \\
\hline Triangular $(\underline{s}, m, \underline{e})$ & 1.5 & 3 & 4.5 & 2.5 & 4 & 5.5 & 3.75 & 12 & 24.75 \\
\hline Triangular $(\bar{s}, m, \bar{e})$ & 1 & 3 & 5 & 2 & 4 & 6 & 3 & 12 & 30 \\
\hline Gaussian $(c, \underline{\sigma})$ & 3 & \multicolumn{2}{|c|}{0.5} & 4 & \multicolumn{2}{|c|}{0.5} & 12 & \multicolumn{2}{|c|}{0.25} \\
\hline Gaussian $(c, \bar{\sigma})$ & 3 & \multicolumn{2}{|c|}{0.8} & 4 & \multicolumn{2}{|c|}{0.8} & 12 & \multicolumn{2}{c|}{0.64} \\
\hline Elliptic $\left(c, d, a_{1}\right)$ & 3 & 2 & 0.8 & 4 & 2 & 0.8 & 12 & See $(16)$ & 0.8 \\
\hline Elliptic $\left(c, d, a_{2}\right)$ & 3 & 2 & 1.2 & 4 & 2 & 1.2 & 12 & See $(16)$ & 1.2 \\
\hline
\end{tabular}

even may be better when compared to other more commonly used type-2 MFs.

Extreme learning machine (ELM) is a non-iterative learning algorithm for single-hidden layer feedforward neural networks. Being non iterative, this algorithm has least number of design parameters. Considering the fact that IT2FLSs can be considered as a special single-hidden layer feedforward neural networks, ELM is used to train IT2FLS [16], [17].

An IF-THEN rule in IT2FLS in SLFN structure can be expressed as follows:

$R^{n}:$ IF $x_{1}$ is $\tilde{X}_{1}^{n}$ and $x_{2}$ is $\tilde{X}_{2}^{n}$ and $\cdots$ and $x_{d}$ is $\tilde{X}_{d}^{n}$

THEN $y^{n}(\mathbf{x})=c_{0}^{n}+c_{1}^{n} x_{1}+\cdots+c_{d}^{n} x_{d}, \quad n=1, \cdots, N$

where $x_{i}$ 's stand for the inputs of the system, $R^{n}$ represents the $n^{\text {th }}$ rule of this structure, $N$ is the total number of rules which is taken as equal to 5 , the parameters $c_{i}^{j}$,s denote the consequent part parameters.

The parameters considered for the elliptic type- 2 MFs are uniformly selected numbers whose range are depicted in Table II.

In order to have a comparison with more common type-2 MFs, Gaussian type-2 MFs with uncertain standard deviation parameter are selected. The parameters selected for this type of MF are illustrated in Table III where $\bar{\sigma}=\sigma+\Delta$ and $\bar{\sigma}=\sigma-\Delta$.

The mean value of root mean squared error (RMSE) for IT2FLSs with both type-2 MFs namely Gaussian type-2 MF with uncertain $\sigma$ parameter and elliptic type-2 MF over 100 times of run of the programs are represented in Table IV. As can be seen from this table, the results obtained by elliptic type-2 MF shows significant improvements when compared to the results obtained by Gaussian type-2 MF with uncertain $\sigma$ parameter. The prediction results for the train and test data are illustrated in Figs 10 and 11, respectively. As can be seen from figures, even though the test data seems to be very different from the train data, the prediction is obtained with a high performance.

TABLE III

PARAMETERS CONSIDERED FOR TYPE-2 MFS FOR THE PREDICTION OF FINANCIAL DATASET

\begin{tabular}{|c|c|c|c|}
\hline \multicolumn{2}{|c|}{ Elliptic type-2 MF } & \multicolumn{2}{c|}{ Gaussian type-2 MF } \\
\hline Parameter & Interval & Parameter & Interval \\
\hline$c$ & {$[0,1]$} & $c$ & {$[0,1]$} \\
\hline$d$ & {$[0.6,1.25]$} & $\sigma$ & {$[0,1]$} \\
\hline$a_{1}$ & {$[1,1.1]$} & $\Delta$ & {$[0,0.1]$} \\
\hline$a_{2}$ & {$[0.9,1]$} & \multicolumn{3}{|c}{} \\
\cline { 1 - 2 }
\end{tabular}

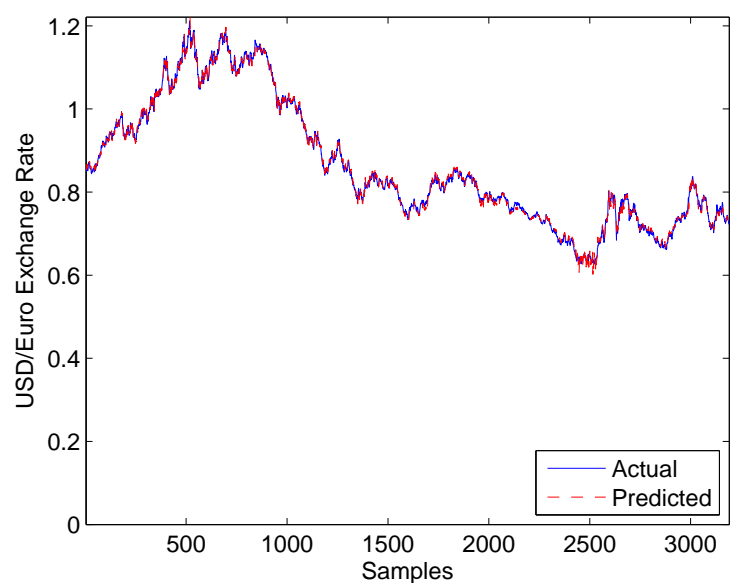

Fig. 10. The results of training data obtained by using IT2FLS with elliptic type-2 MF.

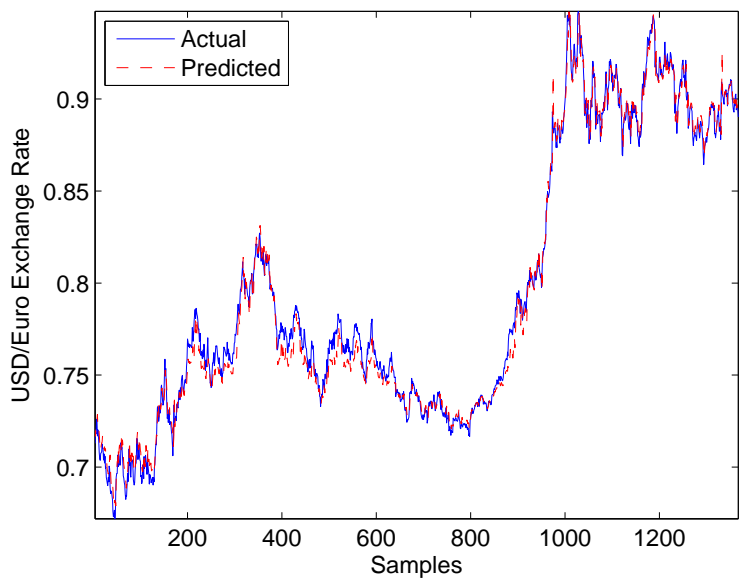

Fig. 11. The results of test data obtained by using IT2FLS with elliptic type-2 MF.

TABLE IV

Mean Value of RMSE

\begin{tabular}{|l|c|c|}
\hline Type-2 MF used & Test & Train \\
\hline Elliptic & 0.0054 & 0.0070 \\
\hline $\begin{array}{l}\text { Gaussian with } \\
\text { Uncertain } \sigma\end{array}$ & 0.0130 & 0.0170 \\
\hline
\end{tabular}

\section{CONCLUSION AND FutURE WORK}

\section{A. Conclusion}

Interval type-2 fuzzy sets are defined by their Footprint of Uncertainty. It is common to create type- 2 fuzzy sets by 


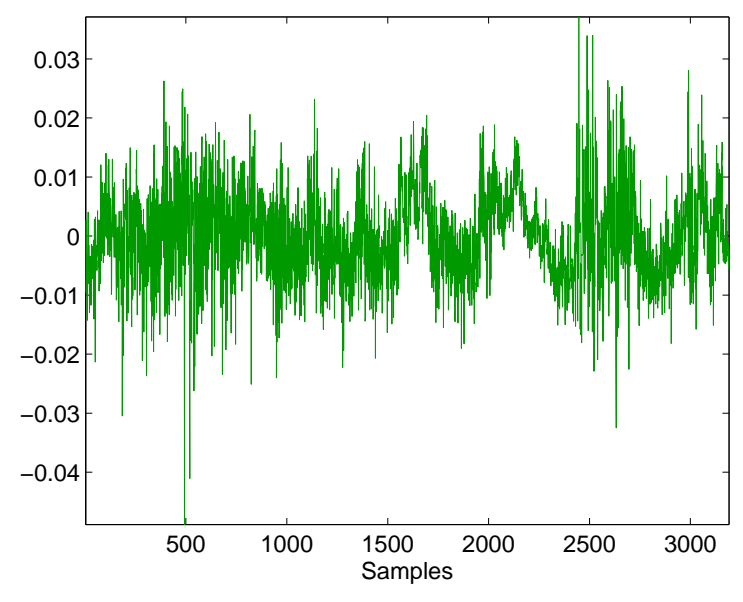

Fig. 12. The results of training data error obtained by using IT2FLS with elliptic type-2 MF.

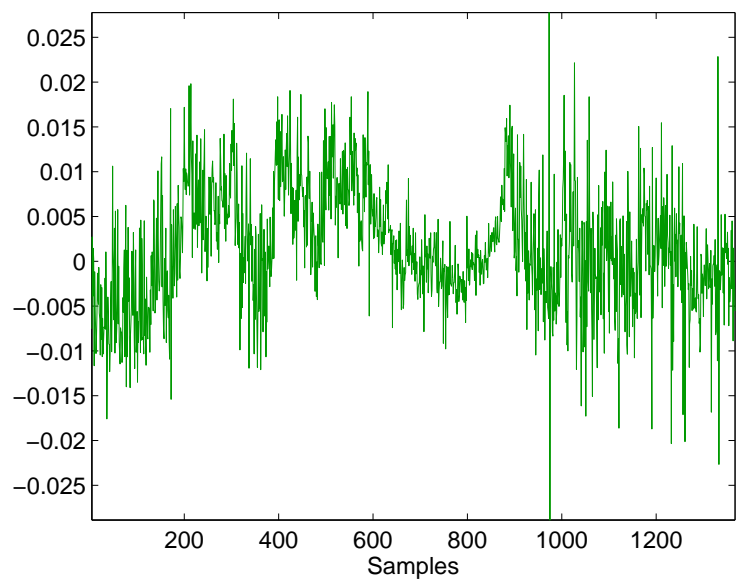

Fig. 13. The results of test data error obtained by using IT2FLS with elliptic type-2 MF.

using triangles, Gaussian or any custom design method. In this paper, we consider a novel elliptic type- 2 membership function and compare and contrast it with the more usual shapes. We have also shown the effects of addition and multiplication operations. In addition to its extra advantages over the existing type-2 fuzzy membership functions such as having decoupled parameters for its support and width, this novel membership function has some similar features to the Gaussian and triangular membership functions in addition and multiplication operations. The aforementioned feature of having decoupled parameters for its support and width allows us to make comprehensive analysis which cannot be done with other membership functions. According to the simulation results, in addition to their various advantages mentioned above, elliptic membership functions have comparable pre- diction results when compared to Gaussian and triangular membership functions.

\section{B. Future work}

Further work will consider their role in real applications modelling human decision making.

\section{REFERENCES}

[1] C.-F. Juang and C.-H. Hsu, "Reinforcement interval type-2 fuzzy controller design by online rule generation and q-value-aided ant colony optimization," Systems, Man, and Cybernetics, Part B: Cybernetics, IEEE Transactions on, vol. 39, no. 6, pp. $1528-1542$, dec. 2009.

[2] C. Juang and Y. Tsao, "A self-evolving interval type-2 fuzzy neural network with online structure and parameter learning," IEEE Trans. on Fuzzy Systems, vol. 16, pp. 1411-1424, 2008.

[3] R. Sepulveda, P. Melin, A. Rodriguez, A. Mancilla, and O. Montiel, "Analyzing the effects of the footprint of uncertainty in type-2 fuzzy logic controllers," Engineering Letters, vol. 13, pp. 138-147, 2006.

[4] L. Zadeh, "Fuzzy sets," Information and Control, vol. 8, pp. 338-353, 1965.

[5] - "The concept of a linguistic variable and its application to approximate reasoning-I," Information Sciences, vol. 8, no. 3, pp. 199 - 249, 1975. [Online]. Available: http://www.sciencedirect.com/science/ article/pii/0020025575900365

[6] J. Rickard, J. Aisbett, and G. Gibbon, "Fuzzy Subsethood for Fuzzy Sets of Type-2 and Generalized Type-n "” Fuzzy Systems, IEEE Transactions on, vol. 17, no. 1, pp. 50-60, Feb 2009.

[7] J. Aisbett, J. Rickard, and D. Morgenthaler, "Type-2 fuzzy sets as functions on spaces," Fuzzy Systems, IEEE Transactions on, vol. 18, no. 4, pp. 841-844, Aug 2010.

[8] M. Khanesar, E. Kayacan, M. Teshnehlab, and O. Kaynak, "Analysis of the noise reduction property of type-2 fuzzy logic systems using a novel type-2 membership function," Systems, Man, and Cybernetics, Part B: Cybernetics, IEEE Transactions on, vol. 41, no. 5, pp. 1395-1406, Oct 2011.

[9] D. Wu, "Twelve considerations in choosing between gaussian and trapezoidal membership functions in interval type-2 fuzzy logic controllers," in Fuzzy Systems (FUZZ-IEEE), 2012 IEEE International Conference on, June 2012, pp. 1-8.

[10] M. Khanesar, E. Kayacan, M. Teshnehlab, and O. Kaynak, "Levenberg marquardt algorithm for the training of type-2 fuzzy neuro systems with a novel type-2 fuzzy membership function," in Advances in Type-2 Fuzzy Logic Systems (T2FUZZ), 2011 IEEE Symposium on, April 2011, pp. 88-93.

[11] M. Khanesar, E. Kayacan, and O. Kaynak, "Optimal sliding mode type-2 tsk fuzzy control of a 2-dof helicopter," in Fuzzy Systems (FUZZ-IEEE), 2015 IEEE International Conference on, Aug 2015, pp. 1-6.

[12] M. Khanesar, M. Teshnehlab, E. Kayacan, and O. Kaynak, "A novel type-2 fuzzy membership function: application to the prediction of noisy data," in Computational Intelligence for Measurement Systems and Applications (CIMSA), 2010 IEEE International Conference on, Sept 2010, pp. 128-133.

[13] S. Coupland and R. John, "An Approach to Type-2 Fuzzy Arithmetic," in Proc. UK Workshop on Computational Intelligence, 2003, pp. 107 114.

[14] W. Blewitt, S.-M. Zhou, and S. Coupland, "Type-2 fuzzy arithmetic," in Proc. FUZZ-IEEE 2007, London, UK, July 2007, pp. 1456 - 1461.

[15] H. Hamrawi and S. Coupland, "Type-2 fuzzy arithmetic using alphaplanes," in Proceedings of IFSA/EUSFLAT 2009, Lisbon, Portugal, 2009, pp. $606-611$.

[16] Z. Deng, K.-S. Choi, L. Cao, and S. Wang, "T2fela: type-2 fuzzy extreme learning algorithm for fast training of interval type-2 tsk fuzzy logic system," IEEE transactions on neural networks and learning systems, vol. 25, no. 4, pp. 664-676, 2014.

[17] S. Hassan, A. Khosravi, and J. Jaafar, "Training of interval type-2 fuzzy logic system using extreme learning machine for load forecasting," in Proceedings of the 9th International Conference on Ubiquitous Information Management and Communication. ACM, 2015, p. 87. 